\title{
A EXPERIÊNCIA DO ESTÁGIO DE DOCÊNCIA
}

Fernanda Barreto Mielke', Agnes Olschowsky²

\begin{abstract}
RESUMO: O presente artigo relata a experiência de uma mestranda acerca do estágio de docência na graduação, desenvolvido na disciplina Enfermagem em Saúde Mental II, do quinto semestre de graduação em Enfermagem, da Universidade Federal do Rio Grande do Sul. O estágio compreendeu os meses de agosto a dezembro de 2007, sendo realizado numa unidade de saúde da família, com duas equipes de saúde da família. A modalidade de atendimento desenvolvida foi visita domiciliar, que busca maior aproximação com a realidade da vida das pessoas. As alunas desenvolveram um trabalho relevante para a unidade em questão, auxiliando na resolução de determinadas situações, oferecendo atendimento de qualidade e ampliando a oferta de serviços aos usuários de saúde mental, visando a reinserção social. A experiência foi de grande valia, integrando diferentes fases do ensino e proporcionando um pensar mais crítico sobre a saúde mental e suas interfaces com as demais áreas da saúde.
\end{abstract}

PALAVRAS-CHAVE: Saúde mental; Enfermagem; Prática do docente de enfermagem.

\section{THE EXPERIENCE OF THE TEACHING TRAINING}

\begin{abstract}
This article reports the experience of the teaching training of graduation students developed in the discipline of Mental Health Nursing II, in the fifth period of the Nursing undergraduate program, at the Federal University of Rio Grande do Sul. The training was developed between August and December , 2007 in a family health unit, with two family health teams. Home visits were performed for to know the reality of the people's life. The students developed a relevant work to the unit in question, assisting in the resolution of certain situations, providing quality care and increasing the supply of services to users of mental health, regarding social reintegration. The experience was of great value, including various stages of education and providing a more critical thinking about mental health and their relations with other health areas.
\end{abstract}

KEYWORDS: Mental health; Nursing; Nursing teacher practice.

\section{LA EXPERIENCIA DE LA PASANTÍA DE DOCENCIA}

RESUMEN: El presente artículo relata la experiencia de una maestreada sobre la pasantía de enseñanza en la graduación, desarrollada en la disciplina de Enfermería en Salud Mental II, del quinto semestre de la graduación en Enfermería, de la Universidad Federal del Rio Grande del Sur. La pasantía comprendió los meses de agosto a diciembre de 2007, siendo realizado en una unidad de salud de la familia, con dos equipos de salud de la familia. La modalidad de atendimiento desarrollada fue visita domiciliar, que busca mayor aproximación con la realidad de vida de las personas. Las alumnas desarrollaran un trabajo relevante para la unidad en cuestión, ayudando en la resolución de determinadas situaciones, ofreciendo atendimiento de calidad y ampliando la oferta de servicios a los usuarios de salud mental, visando la reinserción social. La experiencia fue muy valiosa, integrando diferentes fases de la enseñanza y proporcionando un pensamiento más crítico sobre la salud mental e sus interfaces con las otras áreas de la salud.

PALABRAS CLAVE: Salud mental; Enfermería; Práctica del docente de enfermería.

${ }^{1}$ Enfermeira. Doutoranda do Programa de Pós-Graduação em Enfermagem da Universidade Federal do Rio Grande do Sul-UFRGS. Bolsista CAPES.

${ }^{2}$ Enfermeira. Doutora em Enfermagem Psiquiátrica pela Escola de Enfermagem de Ribeirão Preto da Universidade de São Paulo-EERPUSP. Professora da Escola de Enfermagem e do Programa de Pós-Graduação em Enfermagem da UFRGS.

Autor correspondente:

Fernanda Barreto Mielke

Rua Cel. Fernando Machado 984/1704 - 90010-320 - Porto Alegre-RS

Recebido: 10/07/09

E-mail: fbmielke@gmail.com

Aprovado: 01/08/09 


\section{INTRODUÇÃO}

A formação docente se constitui como uma das principais pautas dos cursos de pós-graduação quando se entende que a prática da docência, o "ser professor" é construído durante a trajetória pessoal e profissional de cada um. Não é possível ensinar sem aprender e vice-versa e, por isso, a docência é uma prática diária, construída e reconstruída a partir dos sujeitos que dela fazem parte ${ }^{(1)}$.

Durante o mestrado acadêmico, desenvolvido no Programa de Pós-Graduação em Enfermagem da Universidade Federal do Rio Grande do Sul-PPGENFUFRGS, a mestranda teve a oportunidade de se aproximar da prática docente, através da disciplina Estágio de Docência na Graduação I, sob a supervisão da professora orientadora.

A disciplina foi cursada pela mestranda no segundo semestre de 2007, com os objetivos de favorecer a aproximação da prática docente, propiciar a participação no processo ensino-aprendizagem na graduação e desenvolver conteúdos que reforcem nossa temática de estudo dentro da linha de pesquisa da pós-graduação.

Assim, propusemos no plano de ensino desenvolver conteúdos acerca do cuidado em saúde mental no modelo psicossocial de atenção, no espaço do território, a partir da realização de visitas domiciliares-VD, ampliando o contato entre saúde mental e atenção básica. O estágio foi realizado na disciplina "Enfermagem em Saúde Mental II", oferecida no quinto semestre do Curso de Graduação em Enfermagem-UFRGS.

O ensino de enfermagem psiquiátrica e saúde mental, de graduação, vem sofrendo transformações em sua práxis. Nas décadas de 1980 e 1990 as disciplinas priorizavam o estudo do normal e do patológico, fragmentando a assistência, concentrando os estágios em hospitais psiquiátricos, dando ênfase às psicopatologias, seguindo a lógica do modo asilar de tratamento ${ }^{(2)}$.

O movimento da Reforma Psiquiátrica Brasileira, iniciado na década de 1970, possibilitou uma nova postura com relação à loucura, compreendendo a pessoa em sua singularidade, objetivando seu bemestar bio-psico-social-cultural-espiritual ${ }^{(3)}$. Por se constituir em um processo político, social, cultural e ideológico, as transformações advindas desse movimento têm acontecido gradativamente, refletindo diretamente no ensino, especialmente no de graduação.
A partir do modo psicossocial de atenção em saúde mental busca-se uma assistência humanizada, usuário-centrada, no intuito de atender as diversas demanda do usuário(4).

Compactuando com a transformação da atenção em saúde mental atualmente tem-se priorizado desenvolver os estágios de graduação em serviços substitutivos ao hospital psiquiátrico, destacando aqueles que compõem a Rede Integral de Atenção em Saúde Mental: atenção básica, Centros de Atenção Psicossocial, ambulatórios especializados, hospital-dia, urgência e emergência psiquiátricas, leitos em hospital geral e serviços residenciais terapêuticos ${ }^{(5)}$.

A proposta atual de ensino deve estar comprometida com o rompimento de barreiras, repensando as práticas assistenciais que perpetuam ações intervencionistas, limitantes e estigmatizantes ${ }^{(6)}$. Desse modo, pauta-se no espaço do território para o desenvolvimento do cuidado e das atividades terapêuticas.

Assim, o presente artigo tem o objetivo de relatar a experiência de docência sob a lógica do modo psicossocial de atenção em saúde mental.

\section{A DISCIPLINA DA GRADUAÇÃO}

A disciplina Enfermagem em Saúde Mental II é oferecida no quinto semestre da graduação. A carga horária a ela destinada é de $150 \mathrm{~h}$, divididas em $20 \mathrm{~h}$ teóricas e 130h teórico-práticas. O corpo docente da disciplina é composto por sete professores, dentre os quais quatro são doutores, um mestre, um especialista e um professor substituto.

As políticas públicas de saúde mental atuais preconizam a transformação do modelo hospitalocêntrico para a atenção integral em saúde, privilegiando espaços substitutivos ao manicômio, enfatizando a inserção social da pessoa em sofrimento psíquico $^{(7)}$. Nesse contexto, o cuidado em saúde mental está pautado em escuta, acolhimento, vínculo, responsabilização, território, equipe multidisciplinar, dando ênfase à subjetividade da pessoa em sofrimento psíquico, atentando para seu cotidiano, sua família e rede social.

Os sete campos de estágios da disciplina da graduação são constituídos dos seguintes serviços: Centros de Atenção Psicossocial (3), hospital geral (1) e atenção básica (3). Não é realizado estágio no hospital psiquiátrico, porque se entende que as práticas desenvolvidas nessa instituição são excludentes, 
contrapondo-se ao modo de atenção em saúde mental que a disciplina apresenta aos alunos. Mas, foi realizada uma visita nesse local para conhecer a realidade desse serviço considerado terapêutico, que está inserido no modelo manicomial e hospitalocêntrico de atenção à saúde mental.

No primeiro dia de aula, há a apresentação dos professores, mestrandos e alunos, do plano de ensino da disciplina e cronograma de atividades do semestre. Esse primeiro contato busca uma aproximação dos alunos com a disciplina, esclarecendo as atividades que serão desenvolvidas ao longo do semestre.

Após os esclarecimentos foi oportunizado o vídeo Estamira, que narrava a história de uma senhora, catadora de lixo, que sofria com uma doença mental. Tal recurso pedagógico teve o objetivo de refletir sobre a proposta assistencial em saúde mental na atualidade, buscando discutir a desmitificação da loucura enquanto incapacitante, perigosa e agressiva.

Seguindo o cronograma da disciplina foram realizadas três aulas expositivo-dialogadas. Esse bloco teórico inicial propõe a aproximação dos graduandos com a saúde mental. A mestranda participou destas aulas como ouvinte, trazendo alguns exemplos práticos em momentos oportunos.

O estágio foi desenvolvido em uma unidade de saúde da família com duas equipes, totalizando 13 profissionais. A Estratégia Saúde da Família representa o novo modelo de atenção em saúde, centrando suas ações na promoção da saúde, pautando-se, especialmente, no acolhimento, no vínculo e na responsabilização pelo cuidado ${ }^{(8)}$.

O grupo de estágio era composto por quatro alunas de graduação, a professora orientadora e a mestranda. O estágio foi realizado nos meses de agosto a dezembro de 2007, em dois dias da semana: nas quintas e sextas-feiras, à tarde. O horário do estágio obedecia ao de funcionamento da unidade de saúde da família, das 13h às $17 \mathrm{~h}$.

Nas quintas-feiras, a unidade realizava a reunião de equipe das $13 \mathrm{~h}$ às $15 \mathrm{~h}$. Neste espaço eram organizadas as atividades da semana, discussão de casos e encaminhamentos. Ao discutir os casos de saúde mental, já eram agendadas as VDs com os agentes comunitários de saúde-ACS e dava-se o retorno às equipes de nossas atividades. Após o término da reunião, nosso grupo se reunia para realizar as supervisões e os seminários.

Nas supervisões realizávamos orientações relacionadas ao manejo e conduta das atividades.
Buscava-se traçar a relação entre teoria e prática, a partir dos seminários temáticos, das aulas teóricas e das VDs realizadas. Também era espaço para discutir sobre as anotações registradas pelas alunas sobre 0 estágio nos diários de campo, que eram lidos pela mestranda. Nesse espaço eram explorados os sentimentos, dúvidas, ansiedades, receios e preconceitos das alunas com o louco e a loucura, com o intuito de facilitar e melhorar o desempenho no manejo com os usuários da saúde mental.

A utilização do diário de campo, instrumento em que são anotadas todas as informações que a pessoa avalie como pertinentes ${ }^{(9)}$, favoreceu a relação professor-aluno entre a mestranda e as alunas, pois a partir das leituras era possível discutir sobre as diferentes dificuldades elencadas por elas de uma maneira geral, buscando minimizá-las.

Os seminários temáticos eram divididos com as alunas de acordo com o tema de interesse, sendo que os dois primeiros e os dois últimos foram realizados pela professora orientadora e pela mestranda. Os temas foram esquizofrenia, transtorno afetivo de humor, substâncias psicoativas, transtornos de ansiedade, transtornos de personalidade, transtornos dissociativos, transtornos alimentares e transtornos sexuais. Os seminários tinham o objetivo de apresentar a psicopatologia, os principais sinais e sintomas, 0 manejo e as possíveis formas de tratamento.

Nas sextas-feiras eram realizadas as VDs, previamente agendadas nas reuniões de equipe com os ACS, que as acompanhavam. Só era dada continuidade às VDs a partir da permissão do usuário e seus familiares. No retorno das VDs, o grupo se reunia para dialogar sobre as atividades desenvolvidas e preparar as evoluções de enfermagem.

A realização da VD permite conhecer a realidade da vida do indivíduo e seu contexto social. Facilita compreender a dinâmica familiar, o relacionamento do usuário com a comunidade e como ocorre sua reinserção social ${ }^{(10,11)}$. Nosso foco nas VDs era exatamente este: a atenção era voltada ao usuário, buscando conhecer seu contexto familiar, sua rede de apoio e como se dava seu contato com a comunidade, em seu território.

Abrir a porta da casa para uma pessoa que não se conhece não é tarefa fácil, pois sentimentos de invasão e perda de privacidade se fazem presentes e era compreensível quando as pessoas nos recebiam na porta, sem convite para entrar. O horário do estágio, 13h, era o da limpeza da cozinha pós-refeição e o da 
conhecida sesta. Antes de sair para as VDs sempre reforçávamos as alunas para que tivessem cuidado ao entrar nas casas, para não constranger a quem nos recebia.

Durante o período do estágio ocorreu uma mudança administrativa no município, contratando as equipes da ESF por uma nova empresa, causando insatisfação em alguns profissionais. Na unidade em que estávamos desenvolvendo o estágio houve desestruturação na organização das equipes, o que resultou no fechamento da unidade por alguns dias.

Com isso, passamos a nos reunir na Escola de Enfermagem, dando continuidade ao estágio. Assistimos ao filme Shine, escolhidos pelas alunas, com o objetivo de refletir sobre o cuidado em saúde mental fora do manicômio. O filme proporcionou compreender aspectos da vida de quem sofre psiquicamente, mostrando que é possível a convivência em comunidade, embora não seja fácil. Na discussão, as alunas apontaram aspectos interessantes sobre o personagem do filme, relacionando com o conteúdo estudado.

Também organizamos, a partir da solicitação das equipes da unidade de saúde, um grupo de saúde mental na comunidade. O primeiro grupo contou com a presença de 14 pessoas entre usuários da saúde mental e seus familiares. Foi um momento rico e de grande aprendizado, porque possibilitou a interação direta com o usuário e seu familiar, escutando os relatos de quem convive com o sofrimento psíquico diariamente. Também demonstrou que o usuário da saúde mental, assim como as demais pessoas, tem uma rotina diária: alguns trabalham e estudam, outros auxiliam nas tarefas domésticas, mantendo relacionamentos intra e extra-familiares, na sua comunidade.

No início as alunas estavam ansiosas, mas no decorrer do grupo foram colocando em práticas os conhecimentos adquiridos na disciplina e o grupo transcorreu de maneira agradável, contando com a participação de todos os presentes. Cremos que participar da atividade do grupo foi importante para as alunas, pois no que se refere à saúde mental, esta modalidade de atenção é frequentemente utilizada nos serviços substitutivos. O grupo terapêutico possibilita a sociabilização e a comunicação, além de ter o objetivo de fazer com que as pessoas em sofrimento psíquico aceitem a tarefa de estudar suas tensões, sintomas e a própria patologia ${ }^{(12)}$. O grupo manteve suas atividades após o final do estágio, contando com a presença das alunas.

No processo avaliativo da disciplina são considerados os seguintes aspectos: a participação, o desenvolvimento do estágio e a realização do trabalho final.

A avaliação do estágio foi realizada em dois momentos. O primeiro momento foi no decorrer do estágio. Reunimo-nos na Escola de Enfermagem e as alunas fizeram a autoavaliação, considerando aspectos como assiduidade; pontualidade; postura ética e profissional; relacionamento com a equipe, usuários, colegas e professoras; estudo; entre outros. Todas as alunas avaliaram que necessitavam estudar mais, pois sentiam dificuldade ao redigir a evolução de enfermagem e no manejo de algumas situações. Essa avaliação durante o estágio proporcionou às alunas a reflexão e melhoria de suas condutas.

A segunda avaliação ocorreu no final do estágio quando elas fizeram novamente a autoavaliação, notando modificações positivas em determinados aspectos de suas condutas, de acordo com os mesmos itens acima citados. Na avaliação final a professora orientadora e a mestranda fizeram a avaliação, verbalmente, de cada uma delas, assim como elas também nos avaliaram. Da primeira para a segunda avaliação foi notório o desenvolvimento das alunas, apresentando modificações positivas com relação à postura profissional frente às equipes e nas VDs, com relação ao manejo com usuários e familiares, na realização das evoluções de enfermagem e melhora nos estudos.

O trabalho final consistia em uma avaliação de família de acordo com o Modelo Calgary de Avaliação de Famílias ${ }^{(13)}$, possibilitando estabelecer a relação entre teoria e prática a partir dos cuidados desenvolvidos pelas alunas. Duas alunas desenvolveram um estudo de caso, pois não obtiveram aprovação dos familiares para realizar a avaliação de família.

As equipes do serviço reconheciam o trabalho desenvolvido pelo grupo de estágio, estando atentas nos momentos de relato de nossas atividades e também às nossas solicitações. Nossa presença na unidade de saúde representava suporte no atendimento em saúde mental, facilitando alguns encaminhamentos e diminuindo a espera pelo atendimento domiciliar.

\section{CONSIDERAÇÕES FINAIS}

A oportunidade de realizar estágios em serviços substitutivos ao hospital psiquiátrico na graduação proporciona ao aluno construir um novo conceito acerca da loucura e da doença mental, valorizando os aspectos 
sociais, culturais, políticos e econômicos imbricados no modo psicossocial de cuidar. $\mathrm{O}$ atendimento baseado na clausura e na exclusão, privando os doentes mentais internados nos grandes manicômios de conviver em sociedade, criou o imaginário de que o louco é perigoso, agressivo e incapaz.

Na medida em que a pessoa em sofrimento psíquico passa a ser atendida no território onde vive, em meio a sua família e seus amigos da comunidade, é possível perceber que o transtorno mental é mais um dado na vida dessa pessoa. Foi essa relação que se buscou construir durante a realização do estágio, ou seja, a experiência docente vivenciada na disciplina tinha o objetivo de, além de propiciar nosso aprendizado, promover a qualidade de vida dos usuários da saúde mental e reinseri-los na vida social e comunitária. Isto só foi possível a partir da compreensão de que a pessoa em sofrimento psíquico necessita de cuidado e atenção como qualquer outro doente crônico e de que tem suas capacidades e limitações, como qualquer indivíduo.

A parceria estabelecida entre ESF e saúde mental ainda é recente na região onde o estágio foi desenvolvido. Por este fato, o trabalho que o grupo desenvolveu nos quatro meses que lá esteve foi importante, auxiliando as equipes na resolução de determinadas situações e melhorando a qualidade do atendimento oferecido aos usuários da saúde mental, buscando observar não apenas a patologia, mas os demais aspectos que compõem a vida cotidiana: a questão social, econômica, física, biológica, espiritual, familiar. Sentimo-nos gratificadas pelo retorno positivo dado pelas equipes ao término de nossa experiência na unidade, porque mostrou que nosso trabalho foi resolutivo em alguma medida.

Finalizando, a experiência de docência foi positiva, integrando diferentes fases do ensino e proporcionando um pensar mais crítico sobre a saúde mental e suas interfaces com as demais áreas da saúde.

\section{REFERÊNCIAS}

1. Freire P. Pedagogia da autonomia: saberes necessários à prática educativa. São Paulo: Paz e Terra; 1996.

2. Kantorski LP, Silva GB. O ensino de enfermagem psiquiátrica e saúde mental - um olhar a partir dos programas das disciplinas. Rev Latino-Am Enferm. 2000 Dez;8(6):27-34.

3. Amarante PDC. Loucos pela vida: a trajetória da reforma psiquiátrica no Brasil. Rio de Janeiro: FIOCRUZ; 1995.
4. Costa-Rosa A. O modo psicossocial: um paradigma das práticas substitutivas ao modo asilar. In: Amarante, Paulo. Ensaios - subjetividade, saúde mental, sociedade. Rio de Janeiro: FIOCRUZ; 2000. p.141-168.

5. Ministério da Saúde (BR). Guia de saúde mental. Governo do Estado do Rio Grande do Sul. Porto Alegre; 2001.

6. Olschowsky A. O ensino de enfermagem psiquiátrica e saúde mental: análise da pós-graduação "Lato Sensu" [tese]. São Paulo (SP): Escola de Enfermagem, Universidade de São Paulo; 2001.

7. Ministério da Saúde (BR). Secretaria de Atenção à Saúde. Coordenação Geral de Saúde Mental. Reforma psiquiátrica e políticas de saúde mental no Brasil. Brasília: Ministério da Saúde. 2005.

8. Ministério da Saúde (BR). Saúde da família: panorama, avaliação e desafios. Brasília: Ministério da Saúde; 2005.

9. Minayo MCS. O desafio do conhecimento: pesquisa qualitativa em saúde. São Paulo: Hucitec, 2007.

10. Reinaldo AMS, Rocha RM. Visita domiciliar de enfermagem em saúde mental: idéias para hoje e amanhã. Rev Eletron Enferm. 2002;4(2):36-41.

11. Botti NCL, Andrade WV. A saúde mental na atenção básica - articulação entre os princípios do SUS e da reforma psiquiátrica. Cogitare Enferm. 2008;13(3):38794.

12. Zimerman DE, Osorio LC. Como trabalhamos com grupos. Porto Alegre: Artmed; 1997.

13. Wright LM, Leahey M. Enfermeiras e famílias: um guia para avaliação e intervenção na família. São Paulo: Roca; 2000. 